\title{
An Energy-saving Operation Strategy for High Speed Trains Based on Genetic Algorithm
}

\author{
Wenting Song ${ }^{1, a^{*}}, \mathrm{Mi} \tan ^{2, b}$, Wenchuan Cai ${ }^{3, c}$ \\ 1,3Beijing Jiaotong University, China \\ ${ }^{2}$ Chongqing University, China \\ *13120222@bjtu.edu.cn
}

\begin{abstract}
Keywords: energy-saving operation strategy; optimization combination technique; genetic algorithm
\end{abstract}

\begin{abstract}
This paper studies an optimization operation strategy for high-speed trains with a purpose of minimizing the running energy consumption. A more comprehensive and energy-saving model based on a priori available information is proposed, especially the geographical factor is first time to be used in establishing energy model. Optimization combination technique is also introduced to solve energy consumption optimization problem of subsections and speed codes for the first time, and genetic algorithm is applied to search speed codes in order to design an energy-saving speed trajectory. Thus there are less decision parameters in the strategy, which reduce the computational complexity and increase the computing speed, making the method become more feasible and favorable for different demands in online operation and offline analysis. To verify the effectiveness of the proposed method, a minimal energy driving on Wuhan-Guangzhou line for CHR-3 is simulated.
\end{abstract}

\section{Introduction}

Today, the total energy consumption of high-speed trains is very huge due to the increase of running speed and the extension of running distance. Therefore, many techniques have been proposed for reducing the operation energy consumption, and many scholars are devoting themselves to study the energy-efficient operation strategy. Kunuhiko was one of the early researchers who explored control strategy for the electric train to research bound state variables of train operation by applying the optimization theory in literature [1]. Howlett derived the condition that an optimal control strategy should be satisfied with minimum value principle when the input parameters changed continuously [2]. However, the continuous control variables were not always consistent with the actual train operation. Scheduling and Control Group put forward a model, in which the discrete throttles were taken as control variables, they also proved four typical phases for energy-saving strategy [3][4].

With the development of intelligent control theory, many scholars had applied fuzzy control, prediction theory and Kawakami had designed a dynamic traction energy-saving strategy for Japan's Shinkansen with prediction control [5]. The work in [6] defined a fuzzy control model to determine economic operation modes of high-speed trains. Nevertheless, the train various rail conditions were not well considered in their studies. In this work, we analyze the rail conditions and put forward the principle of changing acceleration one time on a slope, then an energy-efficient operation model about running energy consumption is established to overcome the shortages of other general strategies. During the optimization process, combination optimization technology is introduced to combine the discretization subsections and speed codes and genetic algorithm is applied to solve the optimization problem, which make the ultimate results be calculated quickly and a running speed curve with minimum energy consumption is obtained. Therefore, it is easy to adjust the energy-efficient reference trajectory for a train when some conditions appear temporarily.

\section{The Energy Model Establishment}

The dynamic description and braking point. The train is regarded as a single particle in this paper 
due to a long rail line. On the basis of analysis for train performance characteristics and line conditions, the motion equations of a high-speed train could be described as

$$
M \frac{d v}{d t}=\mu_{f} F-\mu_{b} B-\left(f_{0}+f_{g}+f_{r}+f_{s}\right) \quad \frac{d t}{d x}=\frac{1}{v}
$$

In the formula, $\mathrm{F}$ and $\mathrm{B}$ are the traction force and braking force respectively, $\mu_{f}$ and $\mu_{b}$ are adjustment coefficient, $\mu_{f} \in(0,1), \mu_{b} \in(0,1)$. $\mathrm{V}$ is the train running speed. $M$ represent the weight of the train, which is changed with the load of a train. $a$ is the train acceleration.

The braking position $x_{b}$ is an important point in the line between the stations, at which braking process is initiated, The braking distance is obtained by a backward integration of the speed trajectory, braking deceleration $a_{b}$ is given according to the braking experience, and the running speed is control at $v_{b}$ before the braking point, so the braking distance $s_{b}$ can be expressed as $s_{b}=v_{b} / 2 a_{b}$, the position of braking point is $x_{b}=s-s_{b}$, $s$ is the distance between two stations.

The Energy Model. Geographical factor is considered in establishing the energy-saving model due to the long distance of high-speed line. Thus a principle of changing acceleration one time on a slope is put forward in this paper, namely the train runs with a constant acceleration or takes coast mode directly at the start of the slope on the basis of the current speed and the target speed, then the speed keeps constant after the train reaches the terminal target speed (before the braking point). In summarize, the acceleration of a train changes one time on a slope at most.

Energy model in traction section. The distance of $K-1$ slopes before the braking point is called traction section, then we analyze the energy consumption. The speed of former slope $v_{i-1}$ and the target speed of current slope $v_{i}$ are known when the distance of changing speed $s_{i l}$ and the distance of constant speed $s_{i 2}$ exist, thus the running time and running distance before changing acceleration on a slope are expressed as follows

$$
t_{i 1}=\int_{v_{i-1}}^{v_{i}} \frac{M}{\mu_{f} F-f_{0}(v)-f(i)-f_{g}(i)} d v \quad s_{i 1}=\int_{v_{i-1}}^{v_{i}} \frac{v}{\mu_{f} F-f_{0}(v)-f(i)-f_{g}(i)} d v
$$

where $f_{g}(i)$ is the gradient resistance and $f(i)$ is the totality of curve resistance and tunnel resistance, the rest running distance is $s_{i 2}=s_{i} s_{i 1}$, and the corresponding running time is $t_{i 2}=s_{i 2} / v_{i}$. Therefore, the total running time and energy consumption on a slope are

$$
t_{i}=t_{i 1}+t_{i 2} \quad E_{i}=\frac{1}{2} M\left(v_{i}^{2}-v_{i-1}^{2}\right)+\left[f_{i}(x)+f(i)\right] s_{i}+t_{i 1} \int_{v_{i-1}}^{v_{i}} f_{0}(v) d v+f_{0}\left(v_{i}\right) v_{i} t_{i 2}
$$

where $f_{0}\left(v_{i}\right.$. ) is the train basic resistance, when $t_{i 2}=0$, the train reaches the target speed at the end of the slope fitly.

Energy consumption model in braking section. The total distance of rest slopes in the railway is called braking section. The train keeps moving at a constant speed until it arrives at braking point in slope $K$, thus the running distance is $s_{k 1}=\sum_{K}^{N} s_{i}-s_{b}$, the corresponding running time and energy consumption are:

$$
t_{k 1}=\frac{s_{k 1}}{v_{k-1}} \quad E_{k 1}=\left[f(k 1)+f_{0}\left(v_{k-1}\right)+f_{g}(k 1)\right] v_{k-1} t_{k 1}
$$

where $f(k 1)$ is the gradient resistance and $f_{g}(k 1)$ is the totality of curve resistance and tunnel resistance of the distance of constant speed on slope $K$. Then train begins to brake with a constant deceleration after it passes the braking point, thus the corresponding running time and storage energy of the rest railway can be expressed as follows

$$
t_{k 2}=\frac{s_{b}}{a_{b}} \quad E_{k 2}=\frac{1}{2} M v_{k-1}^{2}-\left[f_{g}(k 2)+f(k 2)\right] \mathrm{s}{ }_{b}-t_{k 2} \int_{0}^{v_{k-1}} f(\mathrm{v}) d v
$$

where $f_{g}(k 2)$ is the gradient resistance and $f(k 2)$ is the totality of curve resistance and tunnel resistance of the rest railway. In summary, a new energy-saving model for high-speed train between two stations 
is established in combination with the slope consition, the final time and energy consumption are the final time and energy consumption are

$$
T=\sum_{i=1}^{k-1} t_{i}+t_{k 1}+t_{k 2} \quad E=\sum_{i=1}^{k-1} E_{i}+E_{k 1}-E_{k 2}
$$

\section{The Energy-saving Operation Strategy}

The target function and optimization method. Fundamental research of optimization problem lies on the explicit objective function. Based on above analysis, the optimization target function for saving energy is formulated as

$$
\begin{aligned}
\min & E=\sum_{i=1}^{k-1} E_{i}+E_{k 1}-E_{k 2} \\
\text { s.t } \quad v\left(x_{0}\right) & =0, v\left(x_{s}\right)=0, v\left(x_{i}\right)<v_{\lim }{ }^{x_{i}} \\
t\left(x_{0}\right) & =0, T-\Delta t \leq t\left(x_{s}\right) \leq T+\Delta t
\end{aligned}
$$

where $v\left(x_{0}\right)$ and $v\left(x_{s}\right)$ are the speed at the beginning and ending of a line respectively. $v\left(x_{i}\right)$ is the train running speed at $i$ th slope, which should be less than the speed limit $v_{\lim }{ }^{x}, t\left(x_{0}\right)$ and $t\left(x_{s}\right)$ are corresponding to the running time at the start and end position respectively (s), $\mathrm{T}$ is the required running time between successive stations and $\Delta t$ is the allow time error.

Literature [6] introduced a discrete method for MRT systems. In this paper, optimization combination technology will be applied and the discretization method is improved. The optimization process is summarized as follows: the distance is discretized, every slope before the braking point is defined as an independent subsection, then the braking section is defined as the last subsection, so the subsection set is $\left(\mathrm{s}_{1}, \mathrm{~s}_{2}, \mathrm{~s}_{3} \cdots \cdots s_{k}\right)$. Next the speed is discretized as a given set of simple digital codes at the range of limit speed equably, and the set is denoted as $\left(v_{1}, v_{2}, v_{3} \cdots \cdots v_{m-1}, v_{m}\right)$. Finally, the speed code of every subsection is searched through genetic algorithm and various combination sequences for speed codes will be got. The train is assumed to run along each speed sequence and generate the corresponding speed curve, the aim of this paper is to obtain a most energy-efficient speed trajectory.

\section{Optimization Algorithm.}

Population initialization.Define $k$ genes in each chromosome on the base of $k$ discretization subsection, the range of each gene value is real 1 tomcorresponding to $\mathrm{m}$ grade speed codes. The speed is set as $v_{1}$ on the initial acceleration stage and the last subsection due to the traction characteristics and accurate parking, namely coding as 1 , other subsections is stochastic under the upper bound. Based on the above definition, a certain number of chromosomes generate in a random way and constitute the initial population.

The fitness value. The fitness function of an energy-saving strategy is denoted as follow

$$
f=\frac{A}{E+\beta\left|\mathrm{T}-t\left(\mathrm{x}_{s}\right)\right|}
$$

where $E$ is the running energy consumption between successive stations, $A$ is an main adjustment coefficient. $\beta$ is a adjustment coefficient for time error.

The convergence judgment. Output the result when the algorithm reaches the maximum number of iterations or continue the operation.

Genetic manipulation. Choice, The best individual copies directly, then a certain number of individuals are selected by roulette method; Cross, A crossover probability $P c$ is given. Then the single point crossover method is adopted; Mutation, Similarly, a mutation probability $P m$ is given and the mutate chromosomes are determined randomly. And a mutation gene determined in a single point mutation approach, which can be modified for arbitrary value under constraint conditions. 
Update. A new generation of individuals generates after all above operation. Return step 2.

\section{Simulation Verification}

The line between Yingde and Qingyuan in Wuhan-Guangzhou high-speed railway is taken into simulation, which distance is $56 \mathrm{~km}$, running time in the timetable is 16 minutes and the allowed time error is 20 seconds. The optimal operation for EMU CHR3 will be studied. Fig. 1 displays the optimal results, including different speed limits, speed codes and practical running speed. The total energy consumption is $1692.6 \mathrm{kWh}$, running time is 944 seconds and differs with the prescribed time for 16 seconds, which lives up to the punctuality requirement.

In order to analyze the effectiveness of the energy-saving strategy, the energy consumption of a speed trajectory without any optimization is used to compare. Fig. 2 is the speed trajectory of a train, spending the minimum time. The energy consumption sums up to $1964.7 \mathrm{kWh}$ and running time is 865 seconds. It is seen that although the operating time is a little shorter, the energy consumption is bigger, which might not be desirable in practice.

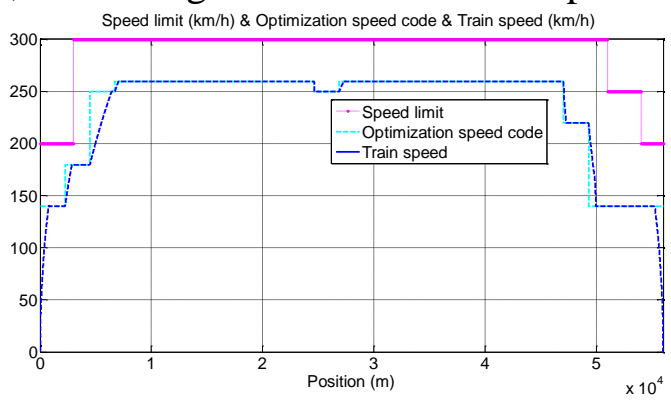

Fig. 1: Trajectory by applying energy-saving strategy.

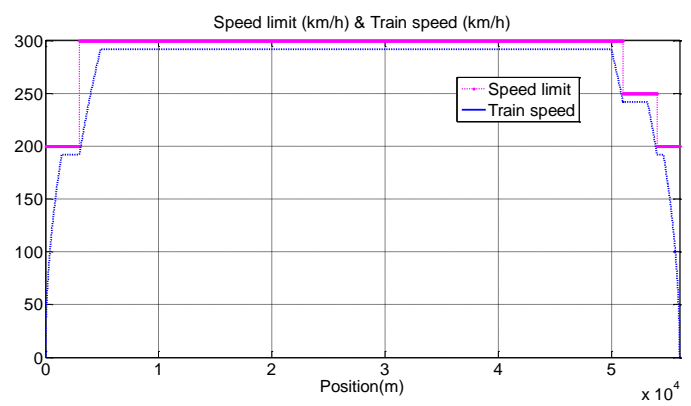

Fig. 2: Trajectory without optimization strategy

\section{Summary}

Aiming at energy-saving control, a new simple and effective train energy model between successive stations is established in this paper, then a new operation strategy on high-speed trains is proposed to optimize the running speed curve with the purpose of reducing energy consumption, which exhibit several attractive advantages: (1) an energy-saving running model is first to establish on the basis of slope analysis; (2) the complicated calculation of some differential equations and nonlinear problems can be avoid by using the established algorithm; (3) less determining parameters are involved in searching the speed code mode to generate the running trajectory; (4) due to the use of discretization for the optimization solution, the overall computing speed is significantly enhanced. Through the simulation of the real transmission line, the results verify the feasibility and effectiveness on energy-saving and punctuality of the proposed method.

\section{References}

[1] I. A. Asnis, A. V. Dmitruk, and N. P. Osmolovskii: Solution of the problem of the energetically optimal-control of the motion of a train by the maximum principle. USSR Comp. Math. Phys. Vol.25, pp.37-44, (1985).

[2] P. G. Howlett, P. J. Pudney: Energy-efficient train control. Springer Press. (1995).

[3] J. X. Cheng, P. G. Howlett: A note on the calculation of optimal strategiesfor the minimization of fuel consumption in the control of trains. IEEE Trans. Autom. Control. Vol.38, pp.1730-1734,(1993).

[4] P. G. Howlett: The optimal control of a train. Annal. Opera. Research. Vol.98, pp.65-87, (2000).

[5] X. Y. Feng: Electric traction drive and control system. Higher Education Press. (2009)

[6] B. R. Ke, C. L. Lin, C. C. Yang: Optimisation of train energy-efficient operation for mass rapid transit systems. IEEE Intel. Transp. Syst. Vol. 6, pp. 58-66, (2012) 